\title{
Local Drug Delivery to Treat Chronic Periodontitis
}

\author{
Mamoora Arslaan, Nasim Karim, Wahab Baksh Kadri, Shama Asghar
}

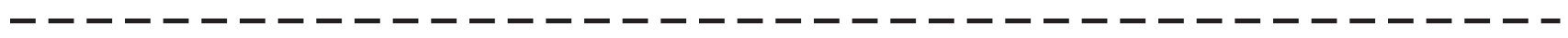

ABSTRACT:

Periodontal disease is a chronic inflammatory disease of periodontium characterized by increased pocket depth, clinical attachment loss, sulcular bleeding, and bone loss. Bacterial plaque bio-film stimulates host derived enzymes and cytokines like TNF $\alpha$, IL-1 and matrix metalloproteases that lead to destruction of periodontium, collagenolytic activity, decreased bone mineral density, intra-bony defects and ultimately bone loss. Chronic periodontitis is managed by conventional and systemic approach, where conventional therapy comprises of scaling and root planning. Mechanical debridement of plaque also requires an adjunct to eradicate the root cause of progressing disease. Hence, worldwide paradigm has shifted towards novel therapies; therefore, local delivery of drug is now preferred due to direct access to target sites with considerably less adverse effects and a better approach to deal with chronic periodontitis. The nano-particle technology to treat periodontitis is still an emerging and promising strategy for the management of disease with the provision of minimal dose, less invasive procedure and clinical efficacy.

Keywords: Conventional therapy, Chronic periodontitis, local drug delivery, plaque, scaling and root planning.

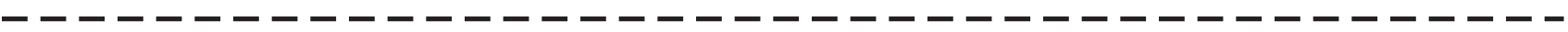

\section{INTRODUCTION:}

Periodontitis is a gradual inflammation of the periodontal apparatus, comprising of gingiva, periodontal ligament and alveolar bone in response to microbial plaque, resulting in annihilation of the periodontium. ${ }^{1}$

The worldwide prevalence of chronic periodontitis among the general adult population is reported to be $30-35 \%{ }^{2}$ whereas according to National Health and Examination Survey III (NHANES III, 1988-1994), 50\% of the adult population in United States, $42 \%$ in Britain, $15.3 \%$ in Portuguese population has been reported in a study. ${ }^{3}$ In 2003; Pakistan estimated $93 \%$ for periodontal disease among 65 year old with higher incidence in the rural population. ${ }^{4,5}$

The alveolar bone, gingiva, periodontal ligament and cementum are the closely related structures that collectively form the periodontium. The periodontal ligament is a fibrous, vascularised connective tissue that traverses the lamina dura, anchors the tooth to the alveolar bone, with the other half

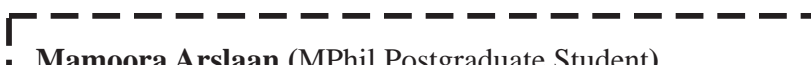

Mamoora Arslaan (MPhil Postgraduate Student)

- Senior Lecturer, Pharmacology Department

I Bahria University Medical and Dental College

I Email: mamooraarslaan2004@gmail.com

Nasim Karim

Professor \& HOD

- Pharmacology Department

I Bahria University Medical and Dental College

Wahab Baksh Kadri

I Principal DentalCollege ,Professor \& HOD

Oral \& Maxillofacial Surgery Department

Bahria University Medical and Dental College

Shama Asghar

Associate Professor \& HOD

I Endodontics Department

I Bahria University Medical and Dental College

| Received: 13-Nov-2019

I Accepted: 05-Mar-2020 attached to the cementum. ${ }^{6}$ The periodontium provides anchorage of tooth to the alveolar bone; provision of a barrier for the underlying structures from the oral microflora and distributed the forces of mastication. ${ }^{7}$

Antoni van Leeuwenhoek, a Dutch researcher, introduced the name of "microbial biofilm" as an adherent layer of microorganisms embedded in extracellular polymeric bacterial matrix. ${ }^{8}$ Amongst pathogens, Treponemadenticola, Porphyromonas gingivalis and L. acidophilus are considered to be responsible for periodontitis. ${ }^{9}$ The disease is initiated by chemotaxis of polymorphonuclear leukocytes (PMN) owing to bacterial lipopolysaccharide (LPS) antigen leading to release of cytokines TNF $\alpha$ and IL1 responsible for the resorption of bone and Matrix metalloproteinases (MMPs) released from fibroblasts that cause destruction of collagen. $\mathrm{TNF} \alpha$ and IL-1 played a vital role in the formation of osteoclasts leading to bone resorption, rapid detachment of gingiva from the tooth surface and ultimately pocket formation. ${ }^{10}$

Evidences are present to demonstrate two-way relation between periodontal and systemic diseases, where both conditions could aggravate each other; such as acute myocardial infarction, infective endocarditis, bacteremia, respiratory diseases, diabetes, alzheimers' disease, pancreatic cancer, cerebral infarction and obesity. Lertpimonchai and colleagues concluded improper tooth brushing techniques and irregular dental visits may lead to the evolution of chronic periodontitis associated with bone loss. ${ }^{11,12}$

Clinical features of chronic periodontitis exhibits morphological changes in gingival tissue like edema, redness, reduced consistency, bleeding on probing, halitosis, loss of clinical gingival attachment, pocket formation, furcation involvement, tooth mobility and alveolar bone loss. ${ }^{13,14}$

According to the severity of the disease, the American Dental Association /American Academy of Periodontology 1999 
guidelines has classified Chronic periodontitis as mild $=>3$ $-<5 \mathrm{~mm}$ periodontal pocket depth (PPD), $1-2 \mathrm{~mm}$ clinical attachment loss (CAL); moderate $==5-<7 \mathrm{~mm} \mathrm{PD}, 3-4$ $\mathrm{mm} \mathrm{CAL}$ and severe $=>7 \mathrm{~mm} \mathrm{PD},=5 \mathrm{CAL}^{.15,16}$

Conventional therapy is the most suggested approach to manage chronic periodontitis, also termed as "Initial therapy" comprising of supra and sub-gingival scaling root planning (SRP) with "adjunctive use of chemical agents." ${ }^{17}$ However, conventional mechanical debridement procedures do not remove all pathogenic bacteria successfully from the subgingival sites, especially the ones found in non-accessible sites namely furcations, grooves, concavities, and deep pockets. Though systemic antibiotics produce beneficial effects in controlling the disease but they have the potential to produce adverse reactions and must be considered equally as their expected benefits. The world has shifted the treatment paradigm from conventional to local delivery system to treat chronic periodontitis. Therefore; this study aimed to assess the various local drug delivery strategies (intra-pocket) in order to provide more potent control of the disease and to avoid systemic adverse effects.

\section{METHODOLOGY:}

The search engines of google, google scholar and pubmed were used for literature search. Keywords and phrases used were chronic periodontitis, intra-pocket administration of drugs, gels, nanoparticles, periodontal fibres, periodontal strips, local delivery systems, adjunctive therapy to scaling and root planning, nanoparticles incorporated with drugs, and administration of drugs sub-gingivally.

The history tracking was from 2014 to 2019. A total of 58 articles were searched and selected for writing the review. Out of these, 2 studies were on non human (rats) and 5 articles had accessibility to abstracts only. 51 articles were relevant to this review. Therefore, 46 articles were selected for writing the review. (Flow Chart).

\section{FLOW CHART}

Filter used literature search period 2014-2019

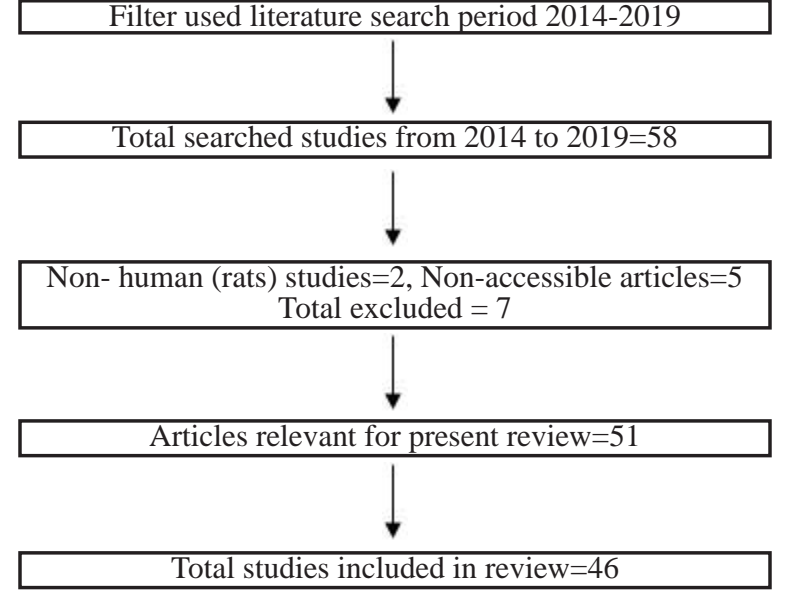

LOCAL DRUG DELIVERY STRATEGIES INTO THE PERIODONTAL POCKET:

Local drug delivery systems have been found beneficial due to direct access to target site which is usually non-responsive to conventional therapy. It also provides good patient compliance, bypasses first-pass metabolism in liver, have longer duration of action, cost effectiveness and safer route of administration. A still better approach is to introduce drug into the periodontal pockets where it actually stays confined, disseminates and is believed to produce better results than the systemic and other local strategies. ${ }^{18}$

Therefore, recommended drug delivery strategies through intrapocket administration are now available for the treatment of periodontitis and administered directly into the periodontal pocket directly. Such as; fibres, periodontal strip, films, gel, microspheres, nano particles, liposomes, nano-fibers, ointments etc.

1: Periodontal Fibers: The Periodontal fibers are the threadlike drug reservoirs that owe to drug delivery systems. They are inserted circumferentially within the periodontal pockets through an applicator. Then, fastened in-situ using an adhesive such as Cyanoacrylate with the provision of sustained release of the drug within the periodontal pocket. ${ }^{19}$ Abeer AK acknowledged that the hollow fibers of cellulose- acetate, infused with tetracycline hydrochloride through which the drug basically disperses out from the reservoir system into the periodontal pockets and helped in eradicating spirochetes effectively from the gingival sulcus. A polymeric fiber device had been manufactured by Johnston \& Co-Workers comprised of alginate with glycerol fibers, cross-linked with positively charged ions of barium incorporated with Ciprofloxacin and Diclofenac Sodium. This drug was $\mathrm{pH}$ dependent and functioned by the process of diffusion at $\mathrm{pH} 4.0$, hence, provides zero-order kinetics. ${ }^{20}$

2: Periodontal Strips: A periodontal strip is an elongated thin, matrix band comprising of a flexible polymerinfused with the drugs dispersed evenly throughout its length with the virtue of securing interproximal space. The periodontal strips presented with noteworthy progress in different clinical parameters by eradicating microbes from the infected periodontal pockets. Since these are non-bio absorbable and removal after therapy is required as it may hamper tissue regeneration at the site. ${ }^{21}$

3: Films: Intra-pocket films are one of the most commonly used local delivery devices for periodontal pockets. The drug incorporated within the matrix of periodontal film spreads evenly throughout the polymer and is released in the infected sites by the process of diffusion or matrix dissolution. ${ }^{22}$ The merits are easy insertion, suitable accommodation within the pockets and patient compliance. Periodontal films comprised of water-insoluble, nondegradable polymersare facilitating the release of drugs by the process of diffusion only whereas others; formed by 
biodegradable soluble polymers release the drugs by the process of diffusion as well as matrix lattices or suspension ${ }^{23}$.Presently,ethylated cellulose films comprising a number of anti-microbials and anti-septics such as Chlorhexidine, Metronidazole, Tetracycline and Minocycline have also been developed so far to produce encouraging results. However; non-biodegradable films have been replaced by biodegradable polymers due to several demerits. ${ }^{24}$

4: Gels: The Gels are semi-solid drug delivery mode and have gained awareness for delivering antimicrobials and other drugs to target the infected sites. This method offers several advantages like easy preparation; management; muco-adhesiveness to non-accessible sites and patient comfort. ${ }^{25}$ While administering gels, the risk of irritation as well as allergies to host at the space of application is comparatively less due to the property of quick eliminating via simple catabolic pathways. Favorable results have also been achieved with the use of a number of organo gels and hydrocolloidal gels for delivery of antimicrobials like Tetracyclines (2.5\%), Metronidazole (25\%), Metronidazole benzoate (40\%), along with the combination of tetracycline and metronidazole benzoate. ${ }^{26}$ The Hydrogels are the hydrophilic polymers comprising of 3D structures in a meshwork of cross-linked structures of hydrogen bonding and ionic bonding. ${ }^{27}$ These type of gels provide structural strength ,easy management at a cellular level and a source of bio-adhesiveness of drug. ${ }^{28}$ Several polymers by the name of poloxamers, PLGA, collagen, D-glucosamine, Acrylic acid, Carbomer are incorporated to formulate a gel to provide an access of drug to the sites where conventional therapy alone cannot achieve desirable results. Gellan gum and lutrol with Ornidazole offered a muco-adhesive gel with the aim of drug release property.The gel used for this purpose is regarded as pseudo plastic, that is liquid at room temperature and less viscous at $34-37^{\circ} \mathrm{C}$. The melting point of the gel is less than the body temperature, permits it to flow without obstruction all the way through the syringe into the periodontal pocket where it turns more viscous. ${ }^{29}$. The Sol-gel transition along with its transformation into semisolid state appears on interaction with GCF due to monoglyceride and triglyceride. The glycerides possess the property of forming liquid crystals, which provides more appropriate controlled release peculiarities when comes into interaction with water. ${ }^{30}$

5: Micro-sphere particles: Microspheres are solid spherical polymeric structures of size ranging from 1-1000 $\mathrm{mm}$ and comprised of a drug which is dispersed throughout the polymeric matrix.These are available in the forms of effusive powders that provides sustained and controlled drug discharge at the specified space. ${ }^{31}$ Presently, there are several nonbiodegradable and bio-degradable materials including polymers from natural and synthetic origin. ${ }^{32}$ Protection of an unstable drug from getting degraded, before and after administration; sustained drug release; patient compliance, controlled therapeutic effects, increased bioavailability and decreased recurrence and intensity of adverse effects are significant benefits of employing micro sphere strategy. ${ }^{33}$ The tetracycline infused micro-sphere particles provided extended release of drug and the antimicrobial activity was observed for more than 30 days against Staphylococcus aureus. ${ }^{34} \mathrm{~A}$ Xanthan polymer infused with rosuvastatin and metformin showed decline in periodontal depth pockets, enhanced the clinical attachment levels by improvisation of gingival attachment as well as promote osteoblastogenesis. ${ }^{35}$

6: Nano particles: The modern era of dentistry have been using so far microparticle-based hydrogels with the property of drug dispersion, which later on found to be affected by their structure. Several factors make the nanoparticles more beneficial over the microspheres, micro particles and emulsion-based delivery systems ${ }^{36}$. These are either dispersed particles or solid particles having size ranging from $10-1000 \mathrm{~nm}$. The drug is suspended, incorporated and encapsulated to a nano particle matrix. ${ }^{37}$ These are dispersible extensively in an aqueous medium, there by offers a controlled release rate and increased stability. The polymerbased nanoparticles are impregnated through micelles polymerization with particle size ranging from $50-180 \mathrm{~nm}$. By virtue of its small size, the nanoparticles can access nonreachable sites like the sub-gingival periodontal spaces and provides an efficient mode of delivery for antimicrobials that can hardly gain access to host cells.$^{38}$

7: Liposomes: Liposomes are the microscopic vesicles of lipids that are categorized as uni lamellar or multilamellar, derived from sphingo lipids, glycolipids, nontoxic surfactants, long-chain fatty acids, cholesterol and even membrane proteins. ${ }^{39}$ They have the advantages of being bio-degradable, biocompatible,non-hazardous, non- immunogenic and highly stable.Simultaneously,few demerits like high cost production, short half-life, micro-leakage have been highlighted. Magnetite nano particles have been recently introduced within the newly developed liposomes like PEG-ylated liposomes and PEG-ylatedmagnetic liposomesranging from anaverage size of $204.3 \mathrm{~nm}$ to $286 \mathrm{~nm}$, respectively,along with an iron component of $4.2 \mathrm{mg} / \mathrm{ml}^{40}$

8: Nano-fibres: Polymeric fibers having diameters in submicrons are known asNano-fibres. ${ }^{41}$ The Nano fibers provide many peculiarities like quite extensive surface area to volume ratio, advanced mechanical performance such as tensile strength, stiffness and rigidity and the flexibility in surface functionalities . In a recent study by Bottino and Co-workers, the effects of Metronidazole and Ciprofloxacin on periodontal pathogens have been assessed by using polydioxanone (PDS) which possesses degradable nano-fibersmatrices. The growth of pathogens like Fusobacteriumnucleatum and Aggregati bacteractinomycetemcomitans was inhibited after utilizing nanofibers comprising of ciprofloxacin while commensals remained unaffected. ${ }^{42}$ In a study of High Performance 
Liquid Chromatography (HPLC), it was revealed that the higher amounts of drug in formulations demonstrated more release over time than those with the minimal amounts, with least dispersion. ${ }^{43}$

9: Ointment: The Minocycline ointment is an absorbableand prolonged drug delivery mode that comprises of $2 \%$ Minocycline $\mathrm{HCl}$ embedded in a matrix ofthe polymers Hydroxyethyl-cellulose, Aminoalkyl-methacrylate and Triacetine ${ }^{44}$ According to a study, $1300 \mathrm{ig} / \mathrm{ml}$ of minocycline has been reported to be available in the pocket spaces for almost an hour, soon after the topical deliverance of 0.05 $\mathrm{ml}$ ointment (1mgof minocycline) which later onreduces to 90ìg/ml after 7 hrs. ${ }^{45}$ Another study has concluded with the results that scaling and root planning in adjunct with ointment application was significantly better than scaling and root planning alone in severe chronic periodontitis owing to periodontal pockets greater than $7 \mathrm{~mm} .{ }^{46}$

The world has shifted the treatment paradigm from the nonresorbable to a varying range of resorbable biodegradable polymers in order to achieve biocompatibility for sustained drug release, reduced frequency of dosing and less risks of developing bacterial resistance. Such strategies augment the effects of scaling and root planning therapy, thereby providing clinical efficacy to treat chronic periodontitis. Advanced clinical knowledge and better understanding of the periodontal disease has led to the introduction of novel drug administration methods which resulted in the innovative designing of targeted delivery systems for the minimization of the systemic adverse effects of anti-microbials and other drugs. These include the periodontal fibers, periodontal strips, periodontal films, chip, micro-particles, nano-particles and nano-fibers.

CONCLUSION: The nano-particle technology to treat periodontitis is still an emerging and promising strategy for the management of disease with the provision of minimal dose, less invasive procedure and clinical efficacy.

\section{REFERENCES:}

1. Srinath,S. Management of periodontal disease with doxycycline: An update. International Journal of Pharmaceutical and Clinical Research. 2015;7(4): 252-55

2. S, R, RD, SH, SR, Khan NAB. Prevalence of chronic periodontitis in an obese population: A preliminary study.BMC Oral Health.2015;15:114. DOI 10.1186/s12903-015-0098-3

3. Al Qahtani NA, Joseph B, Deepthi A, Vijayakumari BK. Prevalence of chronic periodontitis and its risk determinants among female patients in the Aseer Region of KSA. Journal of Taibah University Medical Sciences.2017;12(3): 241-48

4. Altamash M, Klinge B, Engström P.E. Periodontal treatment and $\mathrm{HbA} 1 \mathrm{c}$ levels in subjects with diabetes mellitus. Journal of Oral Rehabilitation 2016; 43:31-8.

5. SalwaMemon ,FarzanaMemon, Hassan Shahid, Diya Ram Khatri, Shoukat Ali Memon. Prevalence of Gingivitis in School Children at Different Government Schools of District Hyderabad. JBUMDC. 2016; 6(4): 236-40
6. Jiang N,Guo W, Chen M,Zheng Y, Zhou J, Kim SG etal. Periodontal ligament and alveolar bone in health andadaptation: tooth movement. Front Oral Biology. 2016;18:1-8.

7. Katancik JA, Kumarswamy A, BranchMays G, Califano JV. Infections of the Periodontal Apparatus.Head, Neck, and Orofacial Infections,2016;189-202. doi:10.1016/b978-0-32328945-0.00011

8. Jamal M, Tasneem U, Hussain T, Andleeb,S. Bacterial Biofilm: Its Composition, Formation and Role in Human Infections. Journal of Microbiology and Biotechnology.2015. eISSN:2320-3528 p-ISSN:2347

9. ColomboAPV, MagalhaesCB, HartenbachFARR, DoSouto, RM,daSilvaBoghossianCM. Periodontal disease associated biofilm: A reservoir for pathogens of medical importance . Microbial Pathogenesis.2015;94 :27-34

10. ZS, RH,LT, HF, DA, HA et al. Chronic periodontitis case definitions and confounders in periodontal research: A Systematic Assessment. HindawiBioMed Research International.2018. Article ID 4578782,

11. A, S, SAO, J, A. The association between oral hygiene and periodontitis: A systematic review and meta-analysis. International Dental Journal.2017;67(6):332-43.

12. SpasovskiS, BelazelkoskaZ, PopovskaM, StojanovskaAA, RadojkovaNikolovskaV, MuratovskaI et al. Clinical therapeutic effects of the application of doxycycline in the treatment of periodontal disease. Macedonian Journal of Medical Sciences. 2017;4(1):152-57.

13. Nazir MA. Prevalence of periodontal disease, its association with systemic diseases and prevention. International Journal of Health Sciences.2017;11( 2): 72-80

14. Attia MA, Mohamed AD, Alharthi AS, Almutawwif MA, Hamad AA, Alhuthali WM. Severity of chronic periodontitis associated with two major risk factors of periodontal disease in Makkah city. J Dent Oral Care.2017; 3(1): 9- 15. DOI: 10.15436/2379-1705.17.1316

15. Tanwar J, Hungund SA, Dodani K. Nonsurgical periodontal therapy: A review. Journal of Oral Research and Review.2016;8: 39-44.

16. DeekshaJ, TarunG, AmitK, Goyal,Goutam R .Advanced drug delivery approaches against periodontitis. Drug Delivery. 2016; 23(2): 363-77, DOI:10.3109/10717544.2014.935531

17. Shivani P, Jaimini G, Harita N, Lakshmi P, Pranav S. Advanced Local Drug Delivery Approaches for Periodontitis: A Strategic Intervention. A Journal of PharmaceuticalScience.2017;9(1): 4-11

18. PooyaDavoodi, Lai YengLee, QingxingXu , Vishnu Sunil, Yajuan Sun, SiowlingSoh, Chi-HwaWang. Drug delivery systems for programmed and on-demand release. Adv. Drug Delivery Reviews. 2018, 1-35. doi.org/10.1016/ j.addr. 2018.07.002

19. MinghanChi, Manlin Qi, Lan A, Ping Wang, Michael DW, Mary A M . Novel Bioactive and Therapeutic Dental Polymeric Materials to Inhibit Periodontal Pathogens and Biofilms. Int. J. Mol. Sci. 2019; 20: 278; 1-29 doi:10.3390/ijms20020278

20. Abeer AK, Doaa AEI, GehanSK, Ragwa MF . Thiolated alginate-based multiple layer mucoadhesive films of metformin for intra-pocket local delivery: in vitro characterization and clinical assessment.Drug Development and Industrial Pharmacy. 2017;43(1):120-31. 
21. Sah AK, Dewangan M, Suresh PK. Potential of chitosanbased carrier for periodontal drug delivery, Colloids and Surfaces B Biointerfaces.2019;178:185-98 doi.org/10.1016/ j.colsurfb.2019.02.04

22. LiviaN,Alfredo D R, Vincenza DG, VincenzoG, Giovanna D. A New Controlled-Release Material Containing Metronidazoleand Doxycycline for the Treatment of Periodontal and Peri-Implant Diseases: Formulation and In Vitro Testing International Journal of Dentistry.2019;110Article ID 9374607. doi.org/10.1155/2019/9374607

23. Urooj A K, Uzma P, Anshul G, GauravKJ ,Farhan JA. Periodontitis: Progression And Treatment Approaches: A Review. Ejbps. 2018;5(6): 153-69.

24. Qinyuan C, Yang J, Xinjun Y. Hydrogels for Biomedical Applications: Their Characteristics and the Mechanisms behind Them. Gels. 2017;3(6):1-15 doi:10.3390/gels3010006

25. KerongY,QingH,BingpengC, YuhaoZ,KesongZ,Qiang L et al. Antimicrobial hydrogels: promising materials for medical application. International Journal of Nanomedicine. 2017;3:2217-30

26. Tarek A, Bader M. A potential in situ gel formulation loaded with novel fabricated poly(lactide-co-glycolide) nanoparticles for enhancing and sustaining the ophthalmic delivery of ketoconazole. International Journal of Nanomedicine 2017:12(5):1863-75

27. Mihaela V G, Mircea H, DumitruL, Cristina E DP. Flow and Thixotropic Parameters for Rheologica Characterization of Hydrogels. Molecules.2016;21:786:1-17doi:10.3390/ molecules21060786

28. Huberth ARJ, Camila FS, Fernanda LS, Ludiele GM, PâmellaCD, Denildo DM. Local Drug Delivery Systems in the Treatmentof Periodontitis: A Literature Review. Journal of the International Academy of Periodontology.2015.17(3): $82-90$

29. Joshi D, Garg T, GoyalAK, RathG.Advanced drug delivery approaches againstperiodontitis.DrugDelivery.2016;23:2,36377. doi:10.3109/10717544.2014.935531

30. GiulianoE, Paolino D, FrestaM, CoscoD. Mucosal Applications of Poloxamer 407-Based Hydrogels: An Overview. Pharmaceutics. 2018; 10(3):159-83doi:10.3390/ pharmaceutics 10030159

31. JianyuLi ,David JM. Designing hydrogels for controlled drug delivery. Nat Rev Mater. 2016; 1(12):4736-45. doi:10.1038/ natrevmats.2016.71.

32. Mil é naL ,Nikolett K S, Vince A, András J ózsefLaki, IstvánAntal. Microparticles, Microspheres, and Microcapsules forAdvanced Drug Delivery. Sci. Pharm. 2019; 87( 20):131.doi:10.3390/scipharm 8703002

33. Zeeshan S, Shariq N, Zohaib K, Vivek V, HaroonR,Michael G. Biodegradable Materials for Bone Repair and TissueEngineering Applications.Materials.2015; 8: 5744-94; doi:10.3390/ma8095273

34. NourAlhusein ,Ian SB, Michael LB, Albert B , Paul ADB . ElectrospunZein/PCL Fibrous Matrices Release Tetracycline in a Controlled Manner, Killing Staphylococcus aureus Both in Biofilms and Ex Vivo on Pig Skin, and are Compatible with Human Skin Cells. Pharm Res. 2016; 33:237-46. DOI 10.1007/s11095-015-1782-3
35. Dileep P, Kurian IG, PradeepAR. Comparative evaluation of subgingivally delivered $1.2 \%$ rosuvastatinand $1 \%$ metformin gelin treatment of intrabony defects in chronic periodontitis: A randomized controlled clinical trial. Journal of Periodontology.2018;89(11):1318-25.doi:10.1002/jper.170434

36. Vijay M, Kuldeep KB, Asit V, Nishika Y, Sourav T, Kalvatala $S$ et al. Solid lipid nanoparticles: emerging colloidal nanodrug delivery systems. Pharmaceutics.2018; 10(4):191-212. doi:10.3390/pharmaceutics10040191

37. H Lu, J Wang, M Stoller, T Wang, Y Bao, HHao. An Overview of Nanomaterials for Water and Wastewater Treatment. Advances in Materials Science and Engineering . 2016.110.doi.org/10.1155/2016/4964828

38. Jean PD, Bérénice HH, Anne LF, Laurence N, Frédéric N. Self-Assembly of Bilayer Vesicles Made of Saturated Long Chain Fatty Acids. Langmuir, American Chemical Society.2016,32(2):401-10.

39. Alfonso TC, Mario FC, Jaime SS ,Ernesto CN, Rafael J, Pavel A et al. Liposomes Loaded with Cisplatin and MagneticNanoparticles:PhysicochemicalCharacterization, $\mathrm{P}$ harmacokinetics, and In-Vitro Efficacy.Molecules.2018; 23(9): 2272-88. doi:10.3390/molecules23092272

40. Rogalski JJ, Bastiaansen C W M ,Peijs T. Rotary jet spinning review - a potential high yield future for polymer nanofibers, Nanocomposites.2017;3(4):97-121, DOI: 10.1080/20550324. 2017.1393919

41. Muhammad Z, Shariq N, Zohaib K, Masoud V, Sana Z,Bilal $\mathrm{N}$ et al. Potential of ElectrospunNanofibersforBiomedical and Dental Applications.Materials.2016; 9: 73-94

42. .Divya P, Maria TPA, Joshua DE, Malgorzata MK, Richard LG, Marco CB. Triple Antibiotic Polymer Nanofibers for Intracanal Drug Delivery: Effects on Dual Species Biofilm and Cell Function. J Endod. 2016;42(10):106-12

43. Marco CB, Rodrigo AA, Aaron W, Krzysztof K, Karen SG, Richard LG Biodegradablenanofibrous drug delivery systems: Effects of metronidazole and ciprofloxacin on periodontopathogens and commensal oral bacteria. ClinOralInvestig. 2014 ; 18(9): 2151-58.

44. Sara A ,Jaideep M , Geetha A. Minocycline Ointment as a Local Drug Delivery in the Treatment of Generalized Chronic Periodontitis - A Clinical Study. Journal of Clinical and Diagnostic Research. 2016 ; 10(6): ZC15-ZC1

45. DeekshaJ, TarunG, AmitK, Goyal,Goutam R .Advanced drug delivery approaches againstperiodontitis. Drug Delivery. 2016; 23(2): 363-77,DOI:10.3109/10717544.2014.93553

46. Xi C, Guofeng W, Zhihong F, Yan D, Wei Z, Bei L, Shizhu B, Yimin Z. Advancedbiomaterials and their potential applications in the treatment of periodontal disease. Informa Healthcare USA. 2015;28:1829-38.DOI: 10.3109/07388551 .2015 .1035693 